\title{
Extremely strong-coupling superconductivity in artificial two-dimensional Kondo lattices
}

\author{
Y. Mizukami ${ }^{1}$, H. Shishido ${ }^{1 \dagger}$, T. Shibauchi ${ }^{1}$, M. Shimozawa ${ }^{1}$, S. Yasumoto ${ }^{1}$, D. Watanabe ${ }^{1}$, \\ M. Yamashita ${ }^{1}$, H. Ikeda ${ }^{1}$, T. Terashima ${ }^{2}$, H. Kontani ${ }^{3}$ and Y. Matsuda ${ }^{1 \star}$
}

\begin{abstract}
When interacting electrons are confined to low dimensions, the electron-electron correlation effect is enhanced dramatically, which often drives the system into exhibiting behaviours that are otherwise highly improbable. Superconductivity with the strongest electron correlations is achieved in heavyfermion compounds, which contain a dense lattice of localized magnetic moments interacting with a sea of conduction electrons to form a three-dimensional Kondo lattice'. It had remained an unanswered question whether superconductivity would persist on effectively reducing the dimensionality of these materials from three to two. Here we report on the observation of superconductivity in such an ultimately strongly correlated system of heavy electrons confined within a twodimensional square lattice of $\mathrm{Ce}$ atoms (two-dimensional Kondo lattice), which was realized by fabricating epitaxial superlattices ${ }^{2,3}$ built of alternating layers of heavy-fermion $\mathrm{CeColn}_{5}$ (ref. 4) and conventional metal $\mathbf{Y b C o l n}_{5}$. The fieldtemperature phase diagram of the superlattices exhibits highly unusual behaviours, including a striking enhancement of the upper critical field relative to the transition temperature. This implies that the force holding together the superconducting electron pairs takes on an extremely strong-coupled nature as a result of two-dimensionalization.
\end{abstract}

The layered heavy-fermion compound $\mathrm{CeCoIn}_{5}$ has the highest superconducting transition temperature $\left(T_{\mathrm{c}}=2.3 \mathrm{~K}\right)$ among rareearth-based heavy-fermion materials ${ }^{4}$. Its electronic properties are characterized by an anomalously large value of the linear contribution to the specific heat (Sommerfeld coefficient $\gamma \sim 1 \mathrm{~J} \mathrm{~mol}^{-1} \mathrm{~K}^{-2}$ ), indicating heavy effective masses of the $4 f$ electrons, which contribute greatly to the Fermi surface. The tetragonal CeCoIn ${ }_{5}$ crystal structure is built from alternating layers of $\mathrm{CeIn}_{3}$ and $\mathrm{CoIn}_{2}$ stacked along the [001] direction. This compound possesses several key features for understanding the unconventional superconductivity in strongly correlated systems ${ }^{5-7}$. The superconductivity with $d_{x^{2}-y^{2}}$ pairing symmetry ${ }^{8-11}$ which occurs in the proximity of a magnetic instability is a manifestation of magnetic-fluctuation-mediated superconductivity ${ }^{5-7,12}$. A very strong coupling superconductivity, where electron pairs are bound together by strong forces, is revealed by a large specific-heat jump ${ }^{4}$ at $T_{\mathrm{c}}$, representing a steep drop of the entropy below $T_{c}$, and a large superconducting energy gap $\Delta$ needed to break the electron pair ${ }^{9}$. Despite its layered structure, the largely corrugated Fermi surface ${ }^{13}$, three-dimensional-like antiferromagnetic fluctuations in the normal state ${ }^{14}$ and small anisotropy of upper critical field ${ }^{15}$ all indicate that the electronic, magnetic and superconducting properties are essentially three dimensional rather than two dimensional. Therefore, it is still unclear to what extent the three-dimensional nature is essential for the superconductivity of $\mathrm{CeCoIn}_{5}$.

Recently, a state-of-the-art technique has been developed to reduce the dimensionality of the heavy electrons in a controllable fashion by layer-by-layer epitaxial growth of Ce-based materials. Previously, a series of antiferromagnetic superlattices $\mathrm{CeIn}_{3} / \mathrm{LaIn}_{3}$ were successfully grown ${ }^{2}$, but it remains open whether heavy electrons in a single Ce layer forming a two-dimensional Kondo lattice can be superconducting. Here we fabricate multilayers of $\mathrm{CeCoIn}_{5}$ sandwiched by non-magnetic and non-superconducting metal $\mathrm{YbCoIn}_{5}$ (the $\mathrm{Yb}$ ion is divalent in closed-shell $4 f(14)$ configuration) forming an $(n: m) c$-axis oriented superlattice structure, where $n$ and $m$ are the number of layers of CeCoIn ${ }_{5}$ and $\mathrm{YbCoIn}_{5}$ in a unit cell, respectively. Small lattice mismatch between $\mathrm{CeCoIn}_{5}$ and $\mathrm{YbCoIn}_{5}$ offers a possibility of providing an ideal heterostructure. The high-resolution cross-sectional transmission electron microscope (TEM) results (Fig. 1a-c), and distinct lateral satellite peaks in the X-ray diffraction pattern (Fig. 1d), demonstrate the continuous and evenly spaced $\mathrm{CeCoIn}_{5}$ layers, with no discernible interdiffusion even for $n=1$ cases (see Supplementary Fig. S1 for quantitative analysis of interdiffusion by X-ray). The epitaxial growth of each layer with atomic flatness is shown by the streak patterns of the reflection high-energy electron diffraction (Fig. 1e) and atomic force microscopy images (Fig. 1f).

We investigate the transport properties for the $(n: 5)$ superlattices by varying $n$. The resistivity $\rho(T)$ of the $\mathrm{CeCoIn}_{5}$ thin film (Fig. 2a) reproduces well that of bulk single crystals ${ }^{4}$. Below $\sim 100 \mathrm{~K}, \rho(T)$ increases on cooling owing to the Kondo scattering, decreases after showing a peak at around the coherence temperature $T_{\text {coh }} \sim 30 \mathrm{~K}$ and drops to zero at the superconducting transition. The hump structure of $\rho(T)$ at $\sim T_{\text {coh }}$ is also observed in the superlattices but becomes less pronounced with decreasing $n$. The superconducting transition to zero resistance is observed in the superlattices for $n \geq 3$ (Fig. 2b). For $n=2$ and $1, \rho(T)$ decreases below $\sim 1 \mathrm{~K}$, but it does not reach zero. However, when the magnetic field is applied perpendicular to the layers for $n=1, \rho(T)$ increases and recovers to the value extrapolated above $1 \mathrm{~K}$ at $5 \mathrm{~T}$, whereas the reduction of $\rho(T)$ below $1 \mathrm{~K}$ remains in the parallel field of $6 \mathrm{~T}$ (Fig. 2c). The observed large and anisotropic field response of $\rho(T)$ is typical for layered superconductors, demonstrating superconductivity even in the $n=1$ superlattice with a two-dimensional square lattice of Ce atoms. The critical temperature $T_{\mathrm{c}}$ determined by the resistive transition gradually decreases with decreasing $n$ (Fig. $2 \mathrm{~d}$ ). The residual resistivity $\rho_{0}$ of the superlattices is of the same order as $\rho_{0}$ of single-crystalline film (Fig. $2 \mathrm{~d}$ ) and is much lower than $\rho_{0}$ of Yb-substituted CeCoIn ${ }_{5}$ single crystals ${ }^{16}$.

${ }^{1}$ Department of Physics, Kyoto University, Kyoto 606-8502, Japan, ${ }^{2}$ Research Center for Low Temperature and Materials Sciences, Kyoto University, Kyoto 606-8501, Japan, ${ }^{3}$ Department of Physics, Nagoya University, Furo-cho, Nagoya 464-8602, Japan. 'Present address: Department of Physics and Electronics, Osaka Prefecture University, Sakai, Osaka 599-8531, Japan. *e-mail: matsuda@scphys.kyoto-u.ac.jp. 


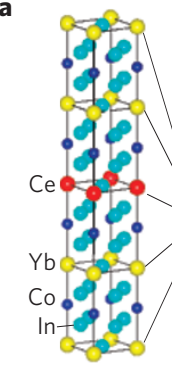

d

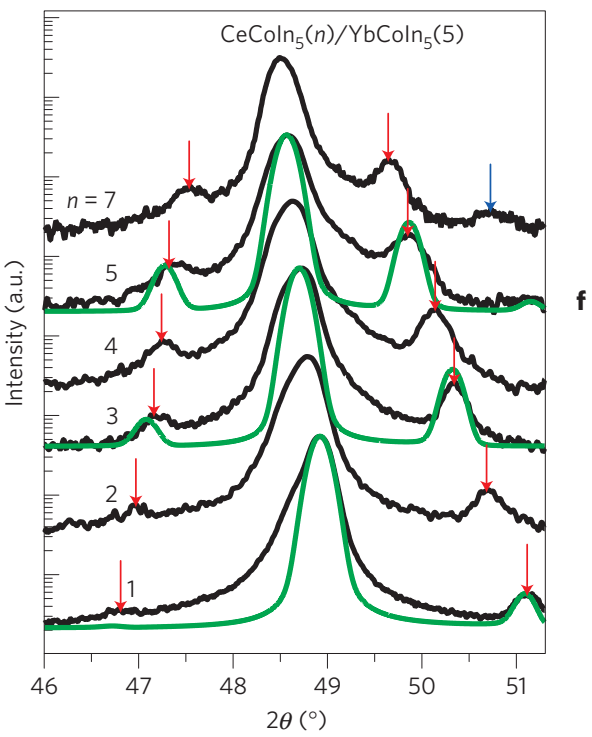

f

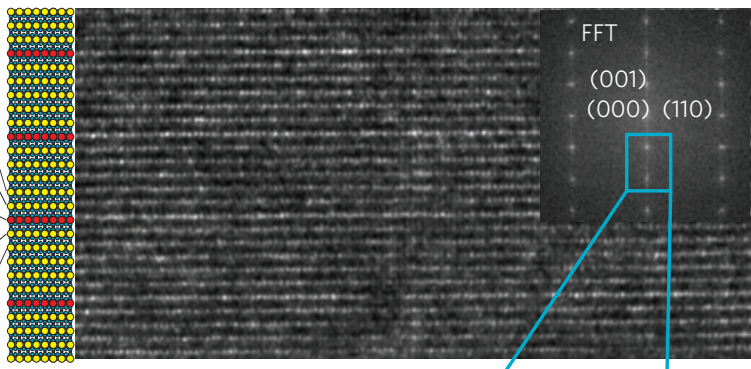

s.
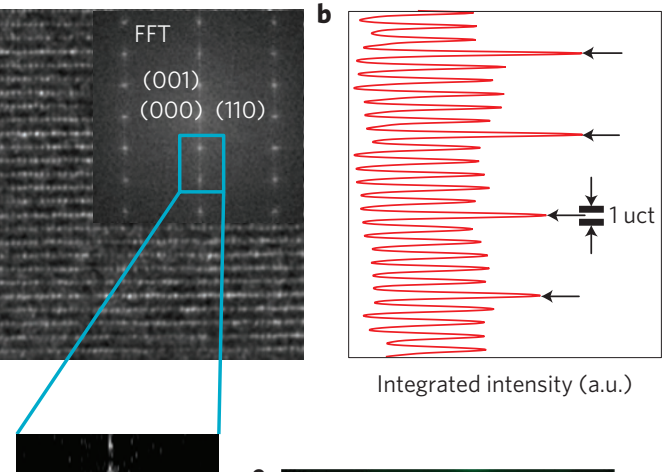

Integrated intensity (a.u.)
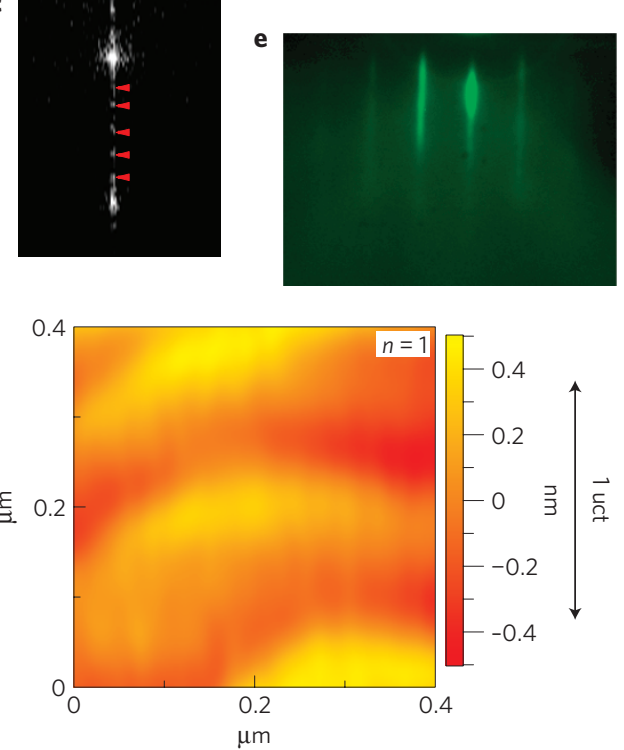

Figure 1 | Epitaxial superlattices $(n: 5)$ of $\mathbf{C e C o l n}_{\mathbf{5}}(\mathbf{n}) /$ YbColn $\mathbf{5}(\mathbf{5})$. a, High-resolution cross-sectional TEM image of $n=1$ superlattice. The bright dot arrays are identified as the Ce layers and the less bright dots are $\mathrm{Yb}$ atoms, which is consistent with the designed superlattice structure in the left panel. b. The intensity integrated over the horizontal width of the image plotted against vertical position indicates a clear difference between the Ce and Yb

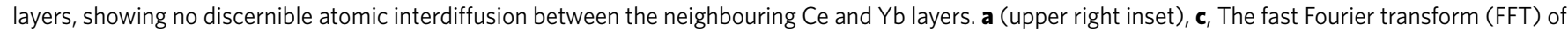

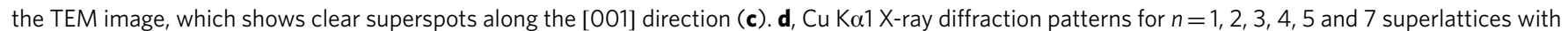
a typical total thickness of $300 \mathrm{~nm}$ show first (red arrows) and second (blue arrow) satellite peaks. The positions of the satellite peaks and their asymmetric heights can be reproduced by the step-model simulations (green lines) ignoring interface and layer-thickness fluctuations ${ }^{29}$ (see also Supplementary Information for the detailed analysis of the satellite peak intensity). e, Streak patterns of the reflection high-energy electron diffraction

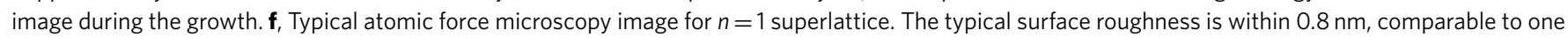
unit-cell thickness (uct) along the $c$ axis of $\mathrm{CeColn}_{5}$.

An important question is whether the superconducting electrons in the superlattices are heavy and if so what their dimensionality is. As shown in Figs 2c, 3a and 4a, the parallel and perpendicular (to the layers) upper critical fields, $H_{\mathrm{c} 2 \|}$ and $H_{\mathrm{c} 2 \perp}$, of the superlattices at low temperature are significantly larger than those in conventional superconductors with similar $T_{c}$. The magnetic field destroys the superconductivity in two distinct ways, the orbital pair-breaking effect (vortex formation) and the Pauli paramagnetic effect, a breaking up of pairs by spin polarization. The zero-temperature value of the orbital upper critical field in perpendicular field $H_{\mathrm{c} 2 \perp}^{\mathrm{orb}}(0)$ reflects the effective electron mass in the plane $m_{a b}^{*}$, $H_{\mathrm{c} 2 \perp}^{\mathrm{orb}}(0) \propto m_{a b}^{* 2}$, and is estimated to be 6,11 and $12 \mathrm{~T}$ for $n=3$, 5 and 7 superlattices from the initial slope of $H_{\mathrm{c} 2 \perp}$ at $T_{\mathrm{c}}$ by the relation $H_{\mathrm{c} 2 \perp}^{\text {orb }}(0)=0.69 T_{\mathrm{c}}\left(-\mathrm{d} H_{\mathrm{c} 2 \perp} / \mathrm{d} T\right)_{T c}$. These magnitudes are comparable with or of the same order as $H_{\mathrm{c} 2 \perp}^{\text {orb }}(0)(=14 \mathrm{~T})$ in bulk single crystal, providing strong evidence for the superconducting 'heavy' electrons in the superlattices. We stress that even a slight deviation of the $f$-electron number from unity leads to a serious reduction of the heavy-electron mass ${ }^{17}$. Moreover, the bandstructure calculation for the $n=1$ superlattice shows that the number of $f$-electrons in each $\mathrm{CeCoIn}_{5}$ layer is very close to unity (Supplementary Information). These facts indicate that the $f$-electron wavefunctions are essentially confined to Ce layers. The magnetic two-dimensionality is shown by estimating the strength of the Ruderman-Kittel-Kasuya-Yosida interaction, an intersite magnetic exchange interaction between the localized $f$ moments, which decays with the distance as $1 / r^{3}$. This interaction between the Ce ions in different layers of $(n: 5)$ superlattices reduces to $(4.568 / 44.716)^{3} \sim 1 \times 10^{-3}$ of that between neighbouring Ce ions within the same layer (see Supplementary Table S1).

The superconducting order parameters in the $\mathrm{CeCoIn}_{5}$ layers of the superlattices are expected to be coupled weakly by the proximity effect through the normal-metal $\mathrm{YbCoIn}_{5}$ layers. The proximity-induced superconductivity in $\mathrm{YbCoIn}_{5}$ layers is expected to be very fragile and destroyed when a weak field is applied ${ }^{18}$. If the thickness of the CeCoIn ${ }_{5}$ layer is comparable to the perpendicular coherence length $\xi_{\perp}\left(\sim 2.1 \mathrm{~nm}\right.$ for CeCoIn $\left.{ }_{5}\right)$, and the separation of superconducting layers $(\sim 3.7 \mathrm{~nm}$ for $(n: 5)$ superlattices) exceeds $\xi_{\perp}$, each CeCoIn ${ }_{5}$ layer acts as a two-dimensional superconductor ${ }^{19}$. This two-dimensional feature is revealed by the diverging $H_{\mathrm{c} 2 \|} / H_{\mathrm{c} 2 \perp}$ of $n=3,5$ and 7 superlattices on approaching $T_{\mathrm{c}}$ (Fig. $3 \mathrm{~b}$ ), in sharp contrast to bulk CeCoIn ${ }_{5}$ (ref. 20), and by a cusplike angular 

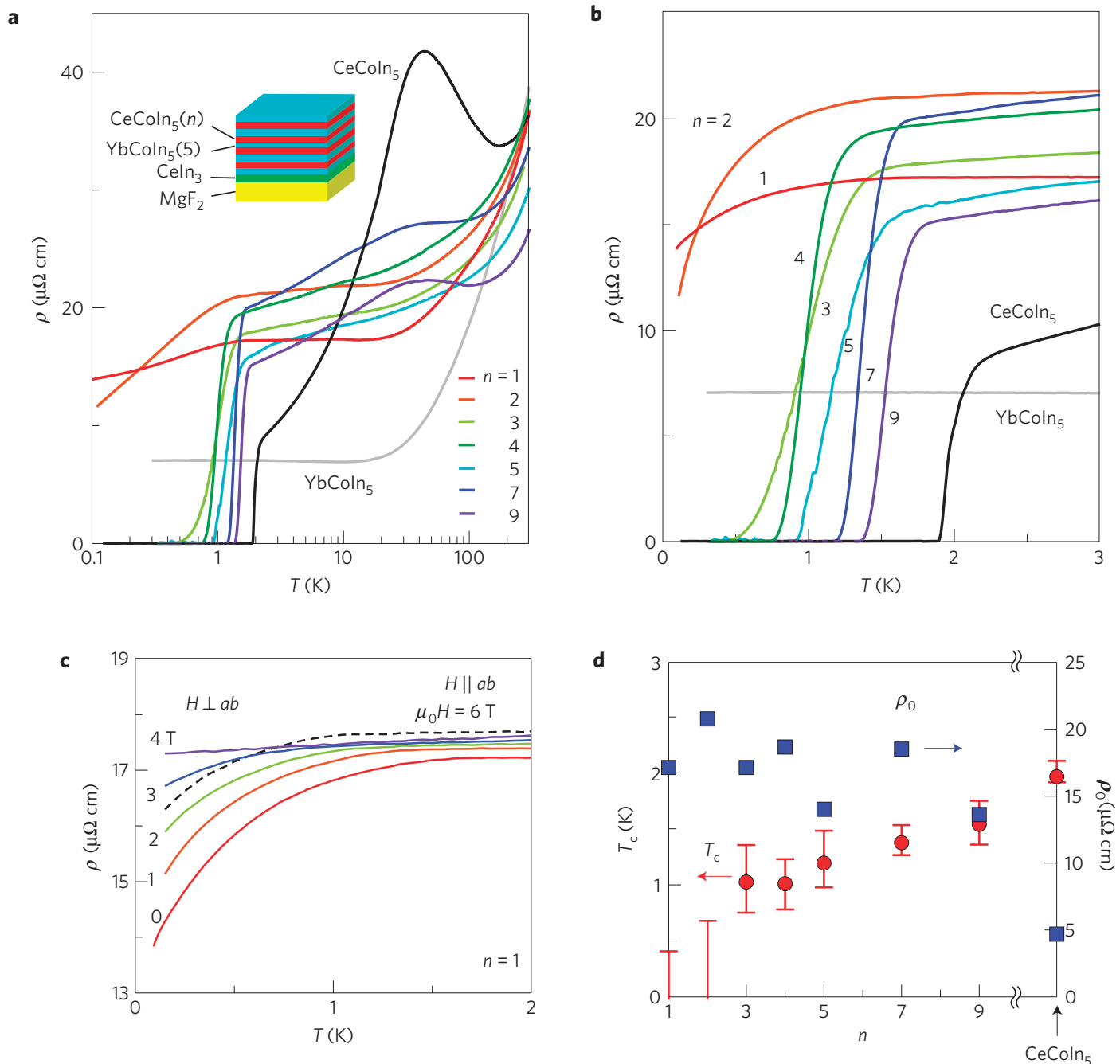

Figure 2 | Superconductivity in superlattices $(\boldsymbol{n}: \mathbf{5})$ of $\operatorname{CeColn}_{\mathbf{5}}(\boldsymbol{n}) / \mathrm{YbColn}_{\mathbf{5}}(\mathbf{5})$. a, Temperature dependence of electrical resistivity $\rho(T)$ for $n=1,2,3,5,7$ and 9, compared with those of 300-nm-thick CeColn ${ }_{5}$ and YbColn ${ }_{5}$ single-crystalline thin films. $\mathbf{b}$, Low-temperature part of the same data as in $\mathbf{a}$. c, $\rho(T)$ for $n=1$ at low temperatures in magnetic field parallel (dotted line) and perpendicular (solid lines) to the $a b$ plane. $\mathbf{d}$, Superconducting transition temperature as a function of $n$ (left axis). The circles are the mid-points of the resistive transition and the bars indicate the onset and zero-resistivity temperatures. The residual resistivity $\rho_{0}$ as a function of $n$ is also shown (right axis).

dependence $H_{\mathrm{c} 2}(\theta)$ near parallel field for the $n=3$ superlattice (Fig. 3c), which is qualitatively different from that expected in the three-dimensional anisotropic-mass model but is well fitted by the model in the two-dimensional limit ${ }^{19}$. On the basis of the above two-dimensional features observed in all electronic, magnetic and superconducting properties, we conclude that the observed heavy-electron superconductivity is mediated most probably by two-dimensional electron-correlation effects.

A fascinating issue is how the two-dimensionalization changes the pairing nature. The fact that $H_{\mathrm{c} 2 \perp}^{\text {orb }}(0)$ estimated from the initial slope of $H_{\mathrm{c} 2 \perp}(T)$ at $T_{\mathrm{c}}$ well exceeds the actual value at low temperatures indicates the predominant Pauli paramagnetic pair-breaking effect even in perpendicular field. Therefore, $H_{\mathrm{c} 2}(\theta)$ at low temperatures is dominated by the Pauli effect in any field direction. This is reinforced by the result that the cusplike behaviour of $H_{\mathrm{c} 2}(\theta)$ becomes less pronounced well below $T_{\mathrm{c}}$ (Fig. 3c), which is the opposite trend to the $H_{\mathrm{c} 2}(\theta)$ behaviour of conventional multilayer systems ${ }^{21}$. In fact, the Pauli-limited upper critical field $H_{\mathrm{c} 2}^{\text {Pauli }}$ given by

$$
H_{\mathrm{c} 2}^{\text {Pauli }}=\sqrt{2} \Delta / g \mu_{B}
$$

where $g$ is the gyromagnetic ratio determined by the Ce crystalline electric field levels, varies smoothly with field direction, consistent with $H_{\mathrm{c} 2}(\theta)$ of the present superlattices at low temperatures. Figure $4 \mathrm{a}$ shows the $H-T$ phase diagram of the superlattices. What is remarkable is that, with decreasing $n, T_{\mathrm{c}}$ decreases rapidly from the bulk value, whereas $H_{\mathrm{c} 2}$ does not exhibit such a reduction, for both field directions. In fact, at low temperatures, $H_{\mathrm{c} 2 \|}$ of $n=5$ and 7 is even larger than that of the bulk. This robustness of $H_{\mathrm{c} 2}$ (and hence of $\Delta$ ) against $n$ reduction indicates that the superconducting pairing interaction is hardly affected by two-dimensionalization. This provides strong evidence that the superconductivity in bulk $\mathrm{CeCoIn}_{5}$ is mainly mediated by two-dimensional spin-fluctuations as in the superlattices with two-dimensional magnetic structure, although the neutron spin resonance mode in bulk $\mathrm{CeCoIn}_{5}$ is observed in the threedimensional $(\pi, \pi, \pi)$ position below $T_{\mathrm{c}}$ (ref. 10).

In sharp contrast to $H_{\mathrm{c} 2}$, the thickness reduction dramatically enhances $H_{\mathrm{c} 2} / T_{\mathrm{c}}$ from the bulk value (Fig. 4b). A comparison to the pressure-dependence results ${ }^{22}$, which represent the increased three-dimensionality, reveals a $T_{c}$ dome and a general trend of enhanced $H_{\mathrm{c} 2} / T_{\mathrm{c}}$ with reduced dimensionality. Through the relation of equation (1), this trend immediately implies a 
a

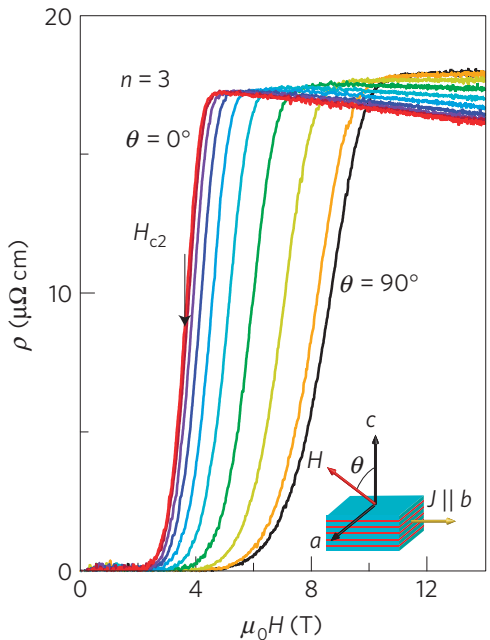

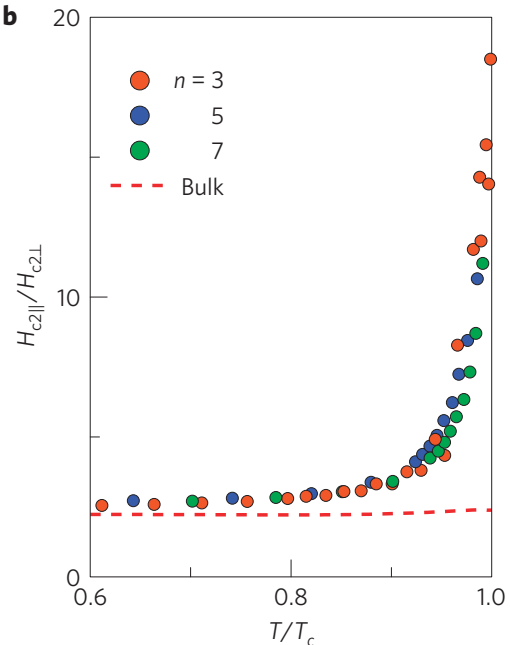

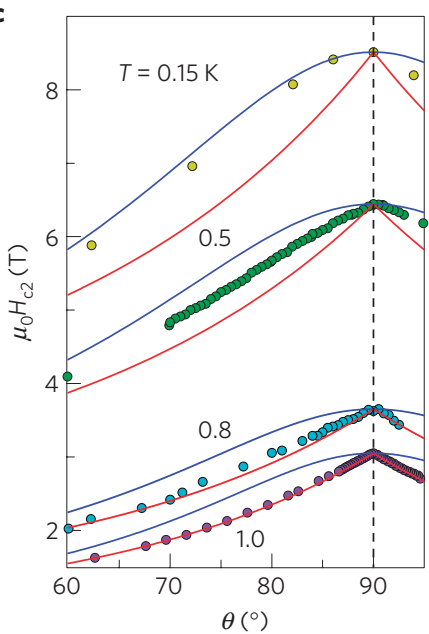

Figure 3 | Superconducting anisotropy in superlattices $(n: 5)$ of $\operatorname{CeColn}_{5}(n) / Y b C o l n_{5}(5)$. a, Magnetic-field dependence of resistivity for $n=3$ superlattice at several field angles from $\theta=0(H \perp a b)$ to $90^{\circ}(H \| a)\left(10^{\circ}\right.$ step) at $T=0.15 \mathrm{~K}$. b. Anisotropy of $H_{\mathrm{c} 2}, H_{\mathrm{c} 2 \|} / H_{\mathrm{c} 2 \perp}$, as a function of reduced temperature $T / T_{\mathrm{c}}$ for $n=3,5$ and 7 superlattices and for the bulk CeColn $5 . \mathbf{c}$, Upper critical field $H_{\mathrm{c} 2}(\theta)$ at several temperatures as a function of field angle $\theta$. $H_{\mathrm{c} 2}$ is determined by the mid-point of the transition except for $1.0 \mathrm{~K}$, where an $80 \%$ resistivity criterion has been used. The solid blue and red lines are the fits to the three-dimensional anisotropic mass model represented as $H_{\mathrm{c} 2}(\theta)=H_{\mathrm{c} 2 \|} /\left(\sin ^{2} \theta+\gamma^{2} \cos ^{2} \theta\right)^{1 / 2}$ with $\gamma=H_{\mathrm{c} 2 \|} / H_{\mathrm{c} 2 \perp}$ and Tinkham's formula $\left|H_{\mathrm{c} 2}(\theta) \cos \theta / H_{\mathrm{c} 2 \perp}\right|+\left[H_{\mathrm{c} 2}(\theta) \sin \theta / H_{\mathrm{c} 2 \|}\right]^{2}=1$ for a two-dimensional superconductor, respectively ${ }^{19}$.

a

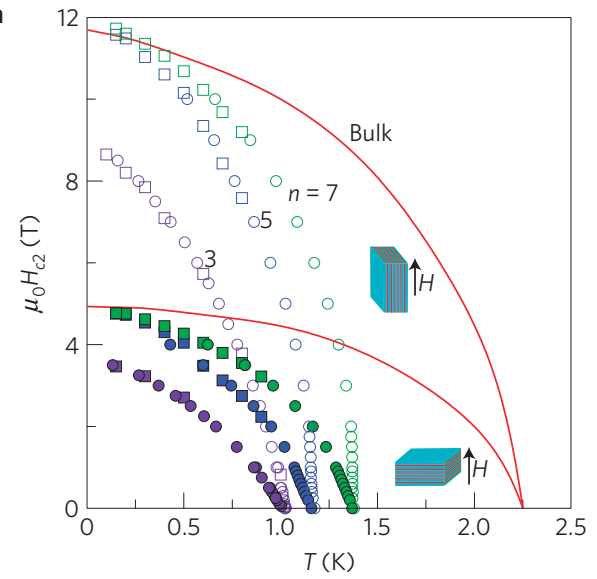

b

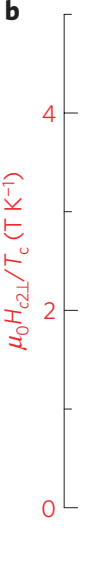

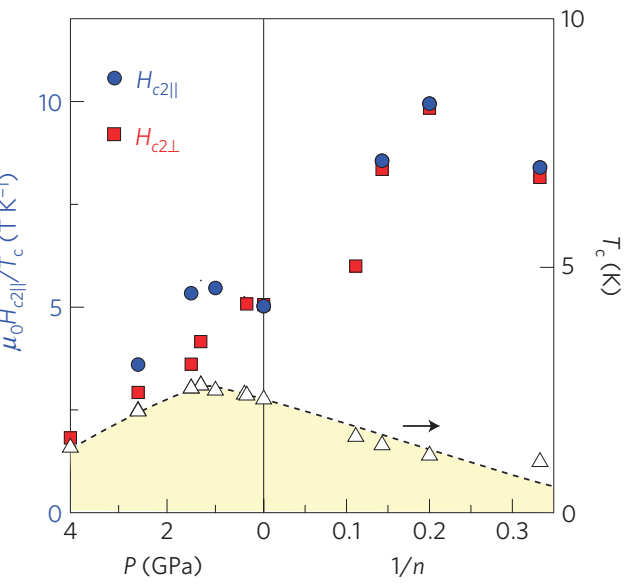

Figure 4 | Superconducting phase diagrams of superlattices $(n: 5)$ of $\operatorname{CeColn}_{5}(n) / \mathrm{YbColn}_{5}(5)$. a, Magnetic-field versus temperature phase diagram of $n=3,5$ and 7 superlattices in magnetic field parallel (open symbols) and perpendicular (closed symbols) to the $a b$ plane, compared to the bulk CeColn $n_{5}$ data. The mid-point of the transition in $\rho(T)$ (circles) and $\rho(H)$ (squares) has been used to evaluate $H_{\mathrm{c} 2}(T)$. b, Superconducting transition temperature, $T_{\mathrm{c}}$ (open triangles), and reduced critical fields $H_{\mathrm{c} 2} / T_{\mathrm{c}}$ in parallel (filled blue circles) and perpendicular (filled red squares) fields as a function of dimensionality parameter $1 / n$ (right panel). The pressure dependence of these quantities ${ }^{28}$ is also shown for comparison (left panel). Note the different scales for parallel (blue) and perpendicular (red) fields.

remarkable enhancement of $\Delta / T_{\mathrm{c}}$ by two-dimensionalization. (As the anisotropy of $H_{\mathrm{c} 2}$ at low temperatures does not depend on $n$, as shown in Fig. 3 b, we assume that the $g$-value of the superlattices deviates little from the bulk value.) We note that the enhanced impurity scatterings cannot be primary origins of the $T_{\mathrm{c}}$ reduction, as these effects do not significantly enhance the $\Delta / T_{\mathrm{c}}$ ratio in $d$-wave superconductors ${ }^{23}$. This is supported by no discernible interdiffusion by TEM results and a $\rho_{0}$ of superlattices of the same order as $\rho_{0}$ of the bulk CeCoIn ${ }_{5}$. The reduction of $T_{\mathrm{c}}$ may be caused by the reduction of density of states in the superlattices, but this scenario is also unlikely because the density-of-states reduction usually reduces the pairing interaction, which results in the reduction of $\Delta$.

Using the reported value of $2 \Delta / k_{\mathrm{B}} T_{\mathrm{c}}=6$ in the bulk single crystal $^{9}, 2 \Delta / k_{\mathrm{B}} T_{\mathrm{c}}$ for the $n=5$ superlattice is estimated to exceed 10 , which is significantly enhanced from the weak-coupling
Bardeen-Cooper-Schrieffer value of $2 \Delta / k_{\mathrm{B}} T_{\mathrm{c}}=3.54$. It has been suggested theoretically that $d$-wave pairing mediated by antiferromagnetic fluctuations in two dimensions can be much stronger than that in three dimensions ${ }^{24-26}$. The striking enhancement of $2 \Delta / k_{\mathrm{B}} T_{\mathrm{c}}$ associated with the reduction of $T_{\mathrm{c}}$, a situation resembling that of underdoped high- $T_{\mathrm{c}}$ cuprates, implies that there seem to be further mechanisms, such as two-dimensional phase fluctuations ${ }^{27}$ and a strong pair-breaking effect due to inelastic scattering ${ }^{28}$. Further investigation, particularly probing electronic and magnetic excitations in the normal and superconducting states, is likely to bridge the physics of highly unusual correlated electrons in the two-dimensional Kondo lattice and in the two-dimensional $\mathrm{CuO}_{2}$ planes of cuprates. The fabrication in a wide variety of nanometric superlattices also opens up a possibility of nanomanipulation of heavy electrons, providing a unique opportunity to produce a new superconducting system and its interface. 


\section{Methods summary}

$\mathrm{CeCoIn}_{5} / \mathrm{YbCoIn}_{5}$ superlattices are grown by the molecular beam epitaxy technique. The pressure of the molecular beam epitaxy chamber was kept at $10^{-7} \mathrm{~Pa}$ during the deposition. The (001) surface of $\mathrm{MgF}_{2}$ with rutile structure $(a=0.462 \mathrm{~nm}, c=0.305 \mathrm{~nm})$ was used as a substrate. The substrate temperature was kept at $550^{\circ} \mathrm{C}$ during the deposition. Atomic layer-by-layer molecular beam epitaxy provides for digital control of layer thickness, which we measure by counting the number of unit cells ${ }^{2}$. Each metal element was evaporated from individually controlled Knudsen cells. 15-unit-cell-thick (uct) $\mathrm{YbCoIn}_{5}$ was grown after $\mathrm{CeIn}_{3}(28 \mathrm{~nm})$ was grown on the (001) surface of substrate $\mathrm{MgF}_{2}$ as a buffer layer. Then $n$-uct CeCoIn 5 layers and $m$-uct $\mathrm{YbCoIn}_{5}$ (typically $m=5$ ) were grown alternately, typically repeated 30-60 times. The deposition rate was monitored by a quartz oscillating monitor and the typical deposition rate was $0.01-0.02 \mathrm{~nm} \mathrm{~s}^{-1}$.

Received 15 June 2011; accepted 8 September 2011; published online 9 October 2011

\section{References}

1. Hewson, A. C. The Kondo Problem to Heavy Fermions (Cambridge Univ. Press, 2003).

2. Shishido, H. et al. Tuning the dimensionality of the heavy fermion compound CeIn ${ }_{3}$. Science 327, 980-983 (2010).

3. Logvenov, G. \& Bozovic, I. Artificial superlattices grown by MBE: Could we design novel superconductors? Physica C 468, 100-104 (2008).

4. Petrovic, C. et al. Heavy-fermion superconductivity in $\mathrm{CeCoIn}_{5}$ at $2.3 \mathrm{~K}$. J. Phys. Condens. Matter 13, L337-L342 (2001).

5. Monthoux, P., Pines, D. \& Lonzarich, G. G. Superconductivity without phonons. Nature 450, 1177-1183 (2007).

6. Sarrao, J. L. \& Thompson, J. D. Superconductivity in cerium- and plutonium-based '115' materials. J. Phys. Soc. Ipn 76, 051013 (2007).

7. Pfleiderer, C. Superconducting phases of $f$-electron compounds. Rev. Mod. Phys. 81, 1551-1624 (2009).

8. Izawa, K. et al. Angular position of nodes in the superconducting gap of quasi-2D heavy-fermion superconductor CeCoIn 5 . Phys. Rev. Lett. 87, 057002 (2001).

9. Park, W. K., Sarrao, J. L., Thompson, J. D. \& Greene, L. H. Andreev reflection in heavy-fermion superconductors and order parameter symmetry in $\mathrm{CeCoIn}_{5}$. Phys. Rev. Lett. 100, 177001 (2008).

10. Stock, C., Broholm, C., Hudis, J., Kang, H. J. \& Petrovic, C. Spin resonance in the $d$-wave superconductor CeCoIn 5 . Phys. Rev. Lett. 100, 087001 (2008).

11. An, K. et al. Sign reversal of field-angle resolved heat capacity oscillations in a heavy fermion superconductor $\mathrm{CeCoIn}_{5}$ and $d_{x^{2}-y^{2}}$ pairing symmetry. Phys. Rev. Lett. 104, 037002 (2010).

12. Mathur, N. D. et al. Magnetically mediated superconductivity in heavy fermion compounds. Nature 394, 39-43 (1998).

13. Settai, R., Takeuchi, T. \& Onuki, Y. Recent advances in Ce-based heavy-fermion superconductivity and Fermi surface properties. J. Phys. Soc. Jpn 76, 051003 (2007).

14. Kawasaki, Y. et al. Anisotropic spin fluctuations in heavy-fermion superconductor $\mathrm{CeCoIn}_{5}$ : In-NQR and Co-NMR studies. J. Phys. Soc. Jpn 72, 2308-2311 (2003).

15. Bianchi, A., Movshovich, R., Capan, C., Pagliuso, P. G. \& Sarrao, J. L. Possible Fulde-Ferrell-Larkin-Ovchinnikov superconducting state in $\mathrm{CeCoIn}_{5}$. Phys. Rev. Lett. 91, 187004 (2003).
16. Shu, L. et al. Correlated electron state in $\mathrm{Ce}_{1-x} \mathrm{Yb}_{x} \mathrm{CoIn}_{5}$ stabilized by cooperative valence fluctuations. Phys. Rev. Lett. 106, 156003 (2011).

17. Rice, T. M. \& Ueda, K. Gutwiller variational approximation to the heavy-fermion ground state of the periodic Anderson model. Phys. Rev. Lett. 55, 995-998 (1985).

18. Serafin, A., Fletcher, J. D., Adachi, S., Hussey, N. E. \& Carrington, A. Destruction of chain superconductivity in $\mathrm{YBa}_{2} \mathrm{Cu}_{4} \mathrm{O}_{8}$ in a weak magnetic field. Phys. Rev. B 82, 140506 (2010).

19. Schneider, T. \& Schmidt, A. Dimensional crossover in the upper critical field of layered superconductors. Phys. Rev. B 47, 5915-5921 (1993).

20. Tayama, T. et al. Unconventional heavy-fermion superconductor $\mathrm{CeCoIn}_{5}$ : Dc magnetization study at temperatures down to $50 \mathrm{mK}$. Phys. Rev. B 65, 180504(R) (2002).

21. Broussard, P. R. \& Geballe, T. H. Critical fields of Nb-Ta multilayers. Phys. Rev. B 35, 1664-1668 (1987).

22. Knebel, G. et al. Competition and/or coexistence of antiferromagnetism and superconductivity in $\mathrm{CeRhIn}_{5}$ and $\mathrm{CeCoIn}_{5}$. Phys. Status Solidi B 247, 557-562 (2010).

23. Sun, Y. \& Maki, K. Impurity effects in $d$-wave superconductors. Phys. Rev. B 51, 6059-6063 (1995).

24. Takimoto, T. \& Moriya, T. Superconductivity and antiferromagnetism in the three-dimensional Hubbard model. Phys. Rev. B 66, 134516 (2002).

25. Arita, R., Kuroki, K. \& Aoki, H. Spin-fluctuation exchange study of superconductivity in two- and three-dimensional single-band Hubbard models. Phys. Rev. B 60, 14585-14588 (1999).

26. Monthoux, P. \& Lonzarich, G. G. Magnetically mediated superconductivity in quasi-two- and three dimensions. Phys. Rev. B 63, 054529 (2001).

27. Emery, V. J. \& Kivelson, S. A. Importance of phase fluctuations in superconductors with small superfluid density. Nature 374, 434-437 (1995).

28. Lee, P. A. \& Read, N. Why is $T_{c}$ of the oxide superconductors so low? Phys. Rev. Lett. 58, 2691-2694 (1987).

29. Fullerton, E. E., Schuller, I. K., Vanderstraeten, H. \& Bruynseraede, Y. Structural refinement of superlattices from x-ray diffraction. Phys. Rev. B 45, 9292-9310 (1992).

\section{Acknowledgements}

We acknowledge discussions with R. Arita, A. V. Chubukov, M. J. Graf, P. A. Lee N. P. Ong, S. A. Kivelson, T. Takimoto and I. Vekhter. This work was supported by KAKENHI from the Japan Society for the Promotion of Science and the Ministry of Education, Culture, Sports, Science and Technology (MEXT) and by a Grant-in-Aid for the Global COE program 'The Next Generation of Physics, Spun from Universality and Emergence' from MEXT.

\section{Author contributions}

The first two authors contributed equally to this work. Y. Matsuda and T.S. planned the experiments, analysed the data and wrote the paper. Y. Mizukami, H.S. and T.T. fabricated the superlattices. M.S., S.Y., D.W. and M.Y. contributed to the measurements. H.I. carried out the band-structure calculation and H.K. analysed the data. All authors critically reviewed the paper.

\section{Additional information}

The authors declare no competing financial interests. Supplementary information accompanies this paper on www.nature.com/naturephysics. Reprints and permissions information is available online at http://www.nature.com/reprints. Correspondence and requests for materials should be addressed to Y. Matsuda 